\title{
Mathematical Model of the Process of Pallet Movement Along the Magnetic Type Centrifugal Brake Roller
}

\author{
Ildar Sharifullin ${ }^{1, *}$, Andrey Nosko ${ }^{1}$, and Yevgeniy Safronov ${ }^{1}$ \\ ${ }^{1}$ Bauman Moscow State Technical University, Faculty of Robotics and Complex Automation, \\ Department of Vertical Transport Systems, 1, 5 2ya Baumanskaya Str., the City of Moscow, 1050056 \\ Russia
}

\begin{abstract}
One of the main elements of safe operation of gravity flow racks used in pallet racks is a brake roller. The most promising design is a centrifugal brake roller of magnetic (eddy current) type. The principle of operation of such rollers is based on the laws of electromagnetic induction and involves the deceleration of a conductor moving in a magnetic field, due to the interaction of eddy currents (or Foucault currents) arising in the volume of the conductor, with an external magnetic field. The mathematical model of the process of pallet movement along a centrifugal brake roller of magnetic type is developed in the article. The equation of the speed of movement of the pallet along the centrifugal brake roller of magnetic type is obtained. Comparative analysis of the speed of movement of a pallet along centrifugal and disc brake rollers of magnetic type is carried out.
\end{abstract}

\section{Introduction}

As a safety device for limiting the speed of movement of pallets with a load (hereinafter referred to as the pallets) in gravity pallet racks $[1,2,3]$, centrifugal friction rollers have found the most widespread use [4]. However, they have a number of disadvantages, the main of which is the wear of the brake facing, and, as a consequence, the change in the braking characteristics of the roller.

The analysis of various designs of brake rollers of gravity conveyors for pallets [4] shows that one of the most promising designs of brake rollers is brake rollers of magnetic (eddy current) type (hereinafter referred to as the MBR), the main advantage of which is non-contact (non-frictional) braking, and, accordingly, no wear on the roller brake facing.

The principle of operation of such rollers is based on the laws of electromagnetic induction and involves the deceleration of a conductor moving in a magnetic field, due to the interaction of eddy currents (or Foucault currents) arising in the volume of the conductor with the external magnetic field [5].

Structurally, MBR can be performed in two versions - either a disk one or centrifugal.

\footnotetext{
* Corresponding author: sharifullin@bmstu.ru
} 
In [6], a mathematical model of the process of pallet movement along the disk MBR is developed. It is found that the speed of movement of the pallet along the disk MBR represents an almost linear dependence, while the slope of the speed graph is determined by the coefficient of magnetic viscosity, the experimental studies of which are described in [7].

If, during the movement of the pallet along the GFR roller bed, the pallet will stop at the place where the disk MBR is installed, then in order to continue moving it must overcome not only the resistance from friction in the bearings of the bearing rollers and the rolling of the pallet along the GFR bearing rollers, which are taken into account when calculating the GFR angle of inclination, but also the braking force of the disk MBR acting on the pallet at any speed of its movement. Thus, when using disk MBR, the GFR angle of inclination should have such a value that takes into account the total resistance to the movement of the pallet, including the braking torque of the disk MBR.

In order to avoid an increase in the GFR angle of inclination, it is proposed to use a centrifugal MBR, in which the braking torque begins to act on the pallet when a certain value of the response speed is reached.

The aim of this work is to develop a mathematical model (hereinafter referred to as the $\mathrm{MM}$ ) of the pallet movement process along the centrifugal MBR.

\section{Object of Study}

\subsection{Design and Operation Description of Centrifugal MBR}

In the GFR, the speed of rotation of the rollers does not exceed $60 \mathrm{rpm}$, which is due to the limitation of the speed of movement of the pallet [4]. In this regard, in the designs of modern brake rollers to increase the braking efficiency, planetary multipliers are used to increase the rotation speed of the brake system to $1000-1500 \mathrm{rpm}$, as well as centrifugal mechanisms, which are widely used in couplings and brakes [8].

Let's consider the design of a centrifugal MBR (Fig. 1), consisting of brake insert 4, on axis 5 of which planetary multiplier 1 , magnetic (eddy current) brake 2 and centrifugal device 3 are installed.

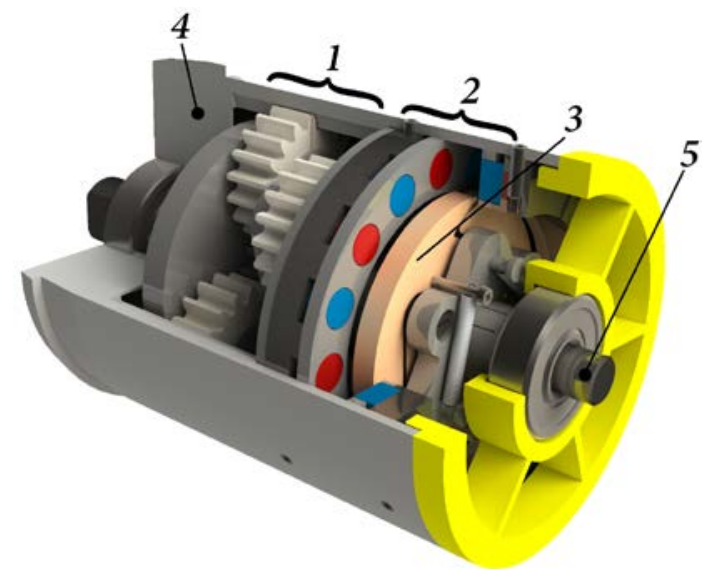

Fig. 1. General view of centrifugal MBR (3D model): 1 - planetary multiplier, 2 - magnetic (eddy current) brake, 3 - centrifugal device, 4 - brake insert, 5 - axis

In the process of movement of the pallet along the centrifugal MBR, it accelerates. This leads to an increase in the rotational speed of brake insert 4 and hub 9 (Fig. 2) of centrifugal device 3 , which is integral with the central gear of the second stage of multiplier 1 . As a 
result, levers 6 of centrifugal device 3 under the influence of centrifugal forces diverge from the center in the radial direction ( Fig. 2, B) and enter the magnetic field of permanent magnets 7 , thereby creating a braking torque. Permanent magnets 7 are arranged with alternating polarity and are rigidly connected by means of an adapter with brake insert 4 . When the speed of brake insert 4 decreases, levers 6 return to their original position under the action of springs 8 (Fig. 2, A).

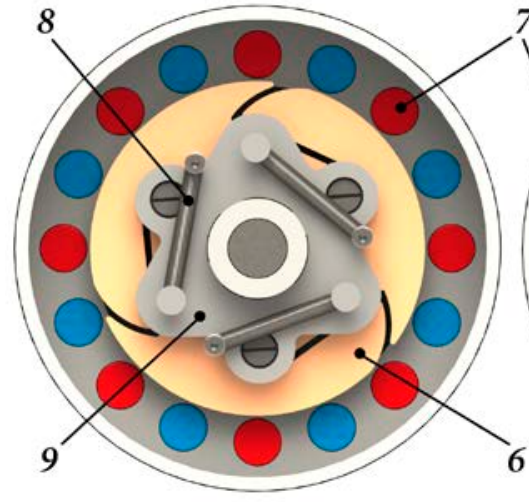

A

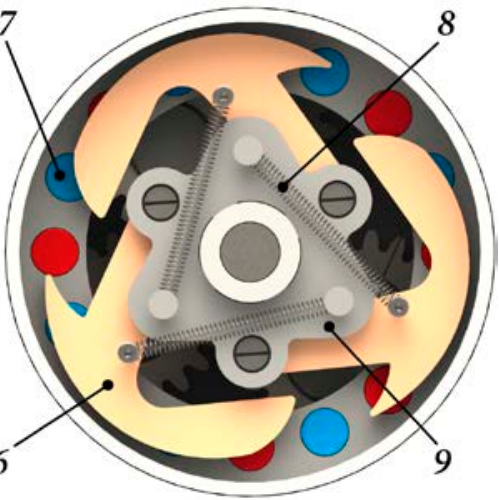

B

Fig. 2. Interaction of the centrifugal device with the eddy current roller brake: A - levers initial position; B - levers final position; 6 - levers; 7 - permanent magnets; 8 - springs; 9 - hub

Levers 6 are made of a material with high conductivity, such as copper or aluminum, and function as conductors. According to the Lorentz force law, eddy currents (Foucault currents) are induced on the surface of the levers and create a moment of resistance to the applied external load (braking torque). As the material of permanent magnets 7, a Nd-Fe-B (neodymium-iron-boron) compound is used. Such magnets have the best magnetic and electrical properties, the service life of which today is 20-25 years and more. They also have a high value of the coercive force, which makes the MBR practically insensitive to the effects of external magnetic fields [9].

In the design of centrifugal MBR, the value of the air gap between magnets 7 and levers 6 remains unchanged, and the braking torque depends on the speed of their relative rotation.

\subsection{Mathematical Model of Calculation}

The processes occurring in the centrifugal MBR when the pallet moves along it, can be divided into two following stages:

1) the acceleration process, in which levers 6 of centrifugal device 3 diverge in the radial direction until they are fully open in the magnetic field;

2) the process of steady motion, in which levers 6 are fully open, a braking torque equal to the driving torque is created, and the pallet enters the motion mode at a constant speed.

During acceleration, when the levers enter the magnetic field, eddy currents begin to form on their surfaces, creating a braking torque that depends not only on the air gap between the levers and permanent magnets, but also on their mutual interference. Estimated calculations have shown that the process of acceleration of the centrifugal MBR does not exceed $5 \%$ of the time of movement of the pallet along the MBR, which makes $6 \ldots 7 \mathrm{sec}$. Therefore, in view of the difficulty of establishing the theoretical dependence of the braking torque on the degree of interference of magnets and levers, the following assumptions can be made: 
- the change in the braking torque during acceleration until the levers are fully open can be neglected due to its short duration;

- the time of the start of the action of the braking torque can be considered this one when the pallet reaches the speed $V_{0}$ of the centrifugal MBR operation, at which the levers are fully open.

With the start of the braking torque, the levers in the end position are disc-shaped. In this case, the speed of movement of the pallet along the centrifugal MBR can be determined in the same way as in the MM calculation of the disk MBR [6] with the corresponding value of the magnetic viscosity coefficient, adding the response speed $V_{0}$ of the centrifugal MBR.

\subsubsection{Centrifugal MBR Response Speed}

The centrifugal MBR response speed $V_{0}$ is the minimum speed that the pallet must have when hitting the centrifugal MBR or when starting to move while stopping on the GFR at the place where the centrifugal MBR is installed before its eddy current brake starts working.

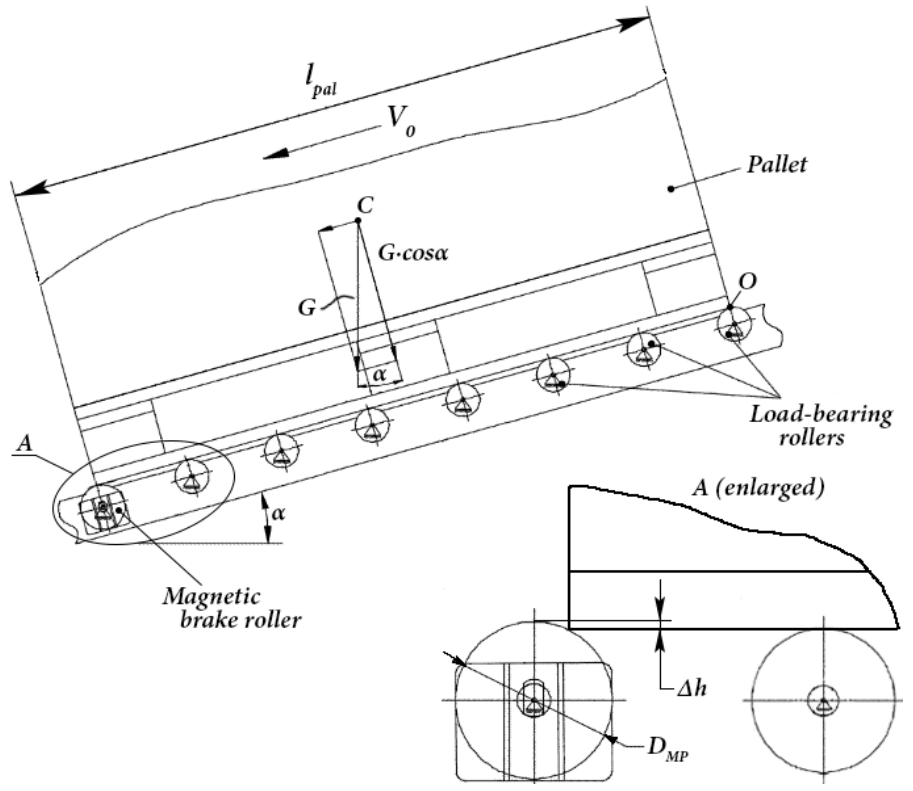

Fig. 3. GFR design model

As a rule, break rollers are installed $\Delta h=1 \ldots 2 \mathrm{~mm}$ higher than the GFR roller bed (Fig. 3 ). In this case, when a pallet moving at a speed $V_{0}$ hits the centrifugal MBR, it rises its front edge by $\Delta h$ relative to point $\mathrm{O}$ (Fig. 3). Let us assume that the mass of the cargo is evenly distributed over the pallet, and the center of gravity of the pallet is located in its geometric center (point C, Fig. 3). Then the center of gravity of the pallet will move by the following value:

$$
\Delta h_{C}=\frac{\Delta h}{2} .
$$

According to the law of conservation of energy when a pallet hits the centrifugal MBR: 


$$
\frac{M \cdot V_{0}^{2}}{2}=G \cos \alpha \cdot \Delta h_{C},
$$

where $M$ - pallet mass, $\mathrm{kg} ; G$ - pallet gravity, N. Taking into account the smallness of the angle of the GFR slope $\alpha=1.7 \ldots 2.8^{\circ}$, the following items can be taken: $\sin \alpha \cong \tan \alpha, \cos \alpha \cong 1$.

Taking into account that $G=M \cdot g$ (where $g=9.81 \mathrm{~m} / \mathrm{sec}^{2}$ - gravity acceleration) and substituting (1) into (2), the centrifugal MBR response speed $V_{0}$ is obtained:

$$
V_{0}=\sqrt{\Delta h \cdot g} .
$$

\subsubsection{Centrifugal device characterization}

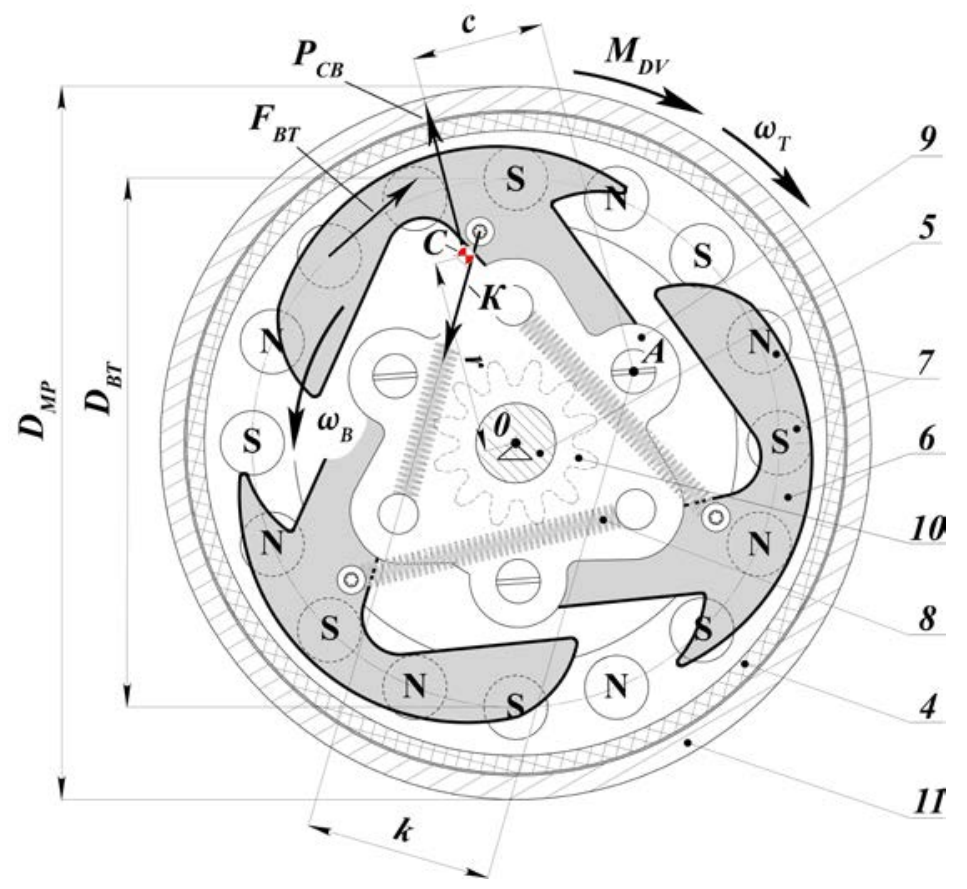

Fig. 4. Centrifugal MBR design model: 4 - brake insert; 5 - axis; 6 - lever; 7 - permanent magnets; 8 - springs; 9 - hub; 10 - multiplier second stage central gear; 11 - shell

To determine the spring force $K$ when the pallet moves along the centrifugal MBR, the following equilibrium equation of forces acting on one lever relative to its axis of rotation (point A, Fig. 4) before the action of the braking force of the eddy current brake, is considered:

$$
\sum M_{A}=P_{C B} \cdot c-2 K \cdot k=0
$$

where $P_{C B}$ - centrifugal force acting on the lever, $\mathrm{N} ; K$ - spring force, $\mathrm{N}$ (since the springs are placed on both sides of the lever, then when they are connected in parallel, the spring forces add up $-2 K) ; c$ - centrifugal arm $P_{C B}, \mathrm{~m} ; k$ - spring forces $K$ arm, m.

From equation (4), the centrifugal force is: 


$$
P_{C B}=\frac{2 K \cdot k}{c}
$$

in addition:

$$
P_{C B}=\frac{m_{P}}{r}\left(\omega_{B} \cdot r\right)^{2}=\frac{m_{P}}{r}\left(\frac{\pi \cdot n_{B}}{30} \cdot r\right)^{2}=\frac{m_{P} \cdot \pi^{2} \cdot n_{B}^{2} \cdot r}{30^{2}}
$$

where $m_{P}$ - lever mass, $\mathrm{kg} ; r$ - distance from the gravity center of the lever (point C, Fig. 4) to the axis of rotation of the hub of the centrifugal device (point O, Fig. 4), m; $\omega_{B}-$ centrifugal device hub angular velocity, $\sec ^{-1} ; n_{B}$ - rotation frequency of the centrifugal device hub, rpm.

Equating (5) and (6), the following is obtained:

$$
K=\frac{m_{P} \cdot \pi^{2} \cdot n_{B}^{2} \cdot r \cdot c}{2 \cdot 30^{2} \cdot k}
$$

Due to the presence of the multiplier, the rotation frequency of the centrifugal device hub is as follows:

$$
n_{B}=u \cdot n_{T}
$$

where $u$ - gear ratio of the centrifugal MBR multiplier; $n_{T}$ - rotational speed of the centrifugal MBR shell, rpm.

Provided there is no pallet slippage along the centrifugal MBR (based on research carried out in [4]), the centrifugal MBR shell rotation frequency $n_{T}$ is as follows:

$$
n_{T}=\frac{60 \cdot V_{0}}{\pi \cdot D_{M P}}
$$

where $D_{M P}$ - centrifugal MBR shell diameter, $\mathrm{m} ; V_{0}$ - centrifugal MBR response speed, $\mathrm{m} / \mathrm{sec}$.

When substituting (3), (8) and (9) in (7), the following formula for determining the elastic force of the centrifugal device spring is obtained:

$$
K=\frac{2 \cdot m_{P} \cdot r \cdot c \cdot \Delta h \cdot g}{k}\left(\frac{u}{D_{M P}}\right)^{2} .
$$

\subsubsection{Pallet speed along the centrifugal MBR}

In [6], a calculated dependence was obtained to determine the speed $V$ of a pallet moving along the disk MBR:

$$
V=\frac{D_{M P}^{2} \cdot g(\tan \alpha-w)}{D_{B T}^{2} \cdot \beta_{1} \cdot n \cdot\left(1+u \cdot \eta_{M P}\right)(1+u)} M
$$

where $V$ - speed of pallet movement along the MBR, $\mathrm{m} / \mathrm{sec} ; w$ - reduced coefficient of resistance to pallet movement on the roller bed of the GFR [10]; $D_{B T} / 2$ - distance from the MBR axis of rotation to the center of permanent magnets, $\mathrm{m} ; \eta_{M P}-\mathrm{MBR}$ coefficient of 
efficiency; $\beta_{1}$-coefficient of magnetic viscosity of a magnet (or a pair of magnets located on both sides of the disc or levers), $\mathrm{N} \cdot \mathrm{sec} / \mathrm{m} ; n$ - number of magnets (or pairs of magnets).

Considering that the levers in the open position (Fig. 2, B) become disk-shaped, and the centrifugal MBR response speed is determined by the formula (3), it is possible to write the following calculated dependence to determine the speed $\mathrm{V}$ of the pallet movement along the centrifugal MBR:

$$
V=\sqrt{\Delta h \cdot g}+\frac{D_{M P}^{2} \cdot g(\tan \alpha-w)}{D_{B T}^{2} \cdot \beta_{1} \cdot n \cdot\left(1+u \cdot \eta_{M P}\right)(1+u)} M .
$$

\section{Results}

As a rule, the centrifugal MBR diameter $D_{M P}$ is chosen based on the GFR and gravity pallet rack design constraints. In action, pipes with diameter $D_{M P}$ equal to 80 or $89 \mathrm{~mm}$ with the wall thickness of $3 \mathrm{~mm}$ made of Steel 3, are most often used in the MBR. In the developed design, (Fig. 1) $D_{M P}=89 \mathrm{~mm}, D_{B H}=83 \mathrm{~mm}$, and based on the analysis carried out in [11], a two-stage multiplier with gear ratio $u=24$ is used. The centrifugal MBR coefficient of efficiency $\eta_{M P}$ can be calculated in a similar manner to the centrifugal friction roller coefficient of efficiency [4]. According to the experimental data [7], the coefficient of magnetic viscosity for the presented design of the centrifugal MBR (Fig. 1) makes $\beta_{1}=$ $0,071 \mathrm{~N} \cdot \mathrm{sec} / \mathrm{m}$ (for a pair of magnets).

The initial data for the calculation of the speed $V$ of the pallet movement along the centrifugal MBR according to the formula (12) are shown in Table 1.

Table 1. Initial data for the calculation of the speed $V$ of the pallet movement along the centrifugal MBR.

\begin{tabular}{|c|c|c|}
\hline Parameter & Value & $\begin{array}{c}\text { Unit of } \\
\text { measurement }\end{array}$ \\
\hline Pallet mass $M$ & $100-1000$ & $\mathrm{~kg}$ \\
\hline Centrifugal MBR shell diameter & 0.089 & $\mathrm{~m}$ \\
\hline $\begin{array}{c}\text { Distance from the axis of rotation of the centrifugal MBR to } \\
\text { the center of permanent magnets } D_{B T} / 2\end{array}$ & 0.033 & $\mathrm{~m}$ \\
\hline $\begin{array}{c}\text { Coefficient of magnetic viscosity } \beta_{I} \\
\text { (for a pair of magnets) }\end{array}$ & 0.071 & $\mathrm{~N} \cdot \mathrm{sec} / \mathrm{m}$ \\
\hline $\begin{array}{c}\text { Reduced coefficient of resistance to pallet movement on the } \\
\text { GFR roller bed } w\end{array}$ & 0.02 & - \\
\hline GFR roller bed slope tan $\alpha$ & 0.04 & - \\
\hline
\end{tabular}

Fig. 5 shows a comparison of the dependences of the speed $V$ of the pallet movement along the two-sided centrifugal MBR, as well as one-sided and two-sided disk MBR (when the MBR is one-sided, magnets are located on one side of the conductor (lever or disk); when it is double-sided, magnets are located on both sides of the conductor). 


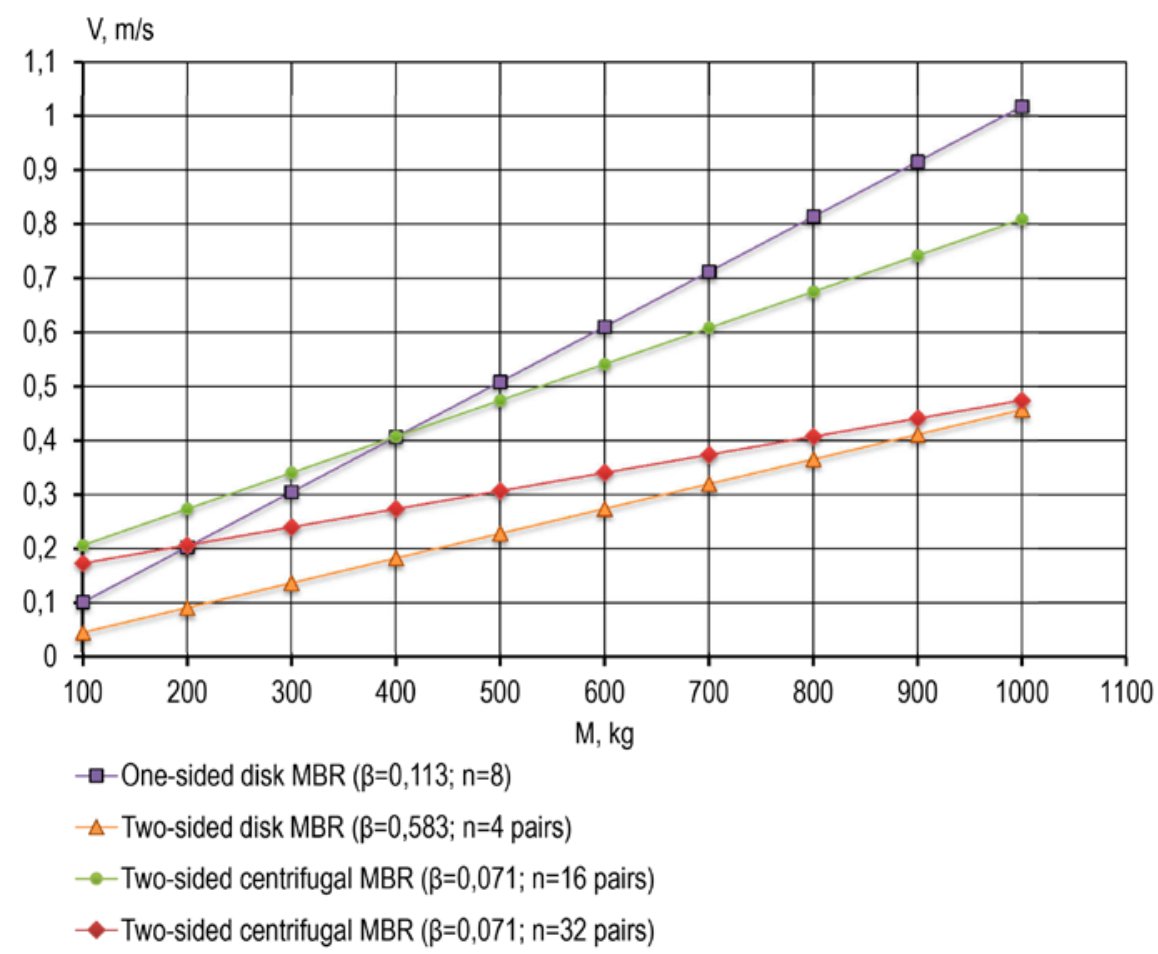

Fig. 5. Dependences of the speed $V$ of the pallet movement along the two-sided centrifugal MBR and one-sided and two-sided disk MBR

As it is seen from Fig. 5, the speed $V$ of movement of a pallet with its mass $M$ along the centrifugal MBR is an almost linear dependence, as well as for the disk MBR, but the response speed $V_{0}$ must be taken into account. At the same time, in terms of braking characteristics, the centrifugal MBR is highly competitive with the disk MBR.

\section{Conclusion}

- The mathematical model of the process of pallet movement along the centrifugal MBR is developed.

- The equation for the speed of the pallet movement along the centrifugal MBR is obtained.

- The braking characteristics of the centrifugal MBR are comparable to those of the disc MBR.

\section{References}

1. N. Boysen, D. Boywitz, F. Weidinger, Deep-Lane Storage of Time-Critical Items: One-Sided versus Two-Sided Access, OR Spectrum, v. 40(4), pp. 1141-1170 (2018)

2. D. Boywitz, N. Boysen, Robust Storage Assignment in Stack- and Queue-Based Storage Systems, Comput. Oper. Res, v. 100, pp. 189-200 (2018)

3. R. Accorsi, G. Baruffaldi, R. Manzini, Design and Manage Deep Lane Storage System Layout. An Iterative Decision-Support Model, Int. J. Adv. Manuf. Technol, v. 92(1-4), pp. 57-67 (2017) 
4. E.V. Safronov, I.A. Sharifullin, A.L. Nosko, Devices for Safe Operation of Pallet Type Gravity Roller Conveyors: Monograph (Universitetskaya kniga, Moscow, 2018) (in Russian)

5. N.I. Kamenskaya, V.A. Sein, M.I. Zvereva, A Study of the Causes of Failure of Permanent Magnets from Cast Hard Magnetic Alloys, Metal Science and Heat Treatment, v. 59, pp. 232-236 (2017)

6. I.A. Sharifullin, A.L. Nosko, E.V. Safronov, Mathematical Model of the Motion Pallet Process on Brake Magnetic Type Roller, The Russian Automobile and Highway Industry Journal, v. 17 (3), pp. 364-373 (2020) (in Russian)

7. I. Sharifullin, A. Nosko, E. Safronov, D. Kirillov, Experimental Study of Eddy Current Braking Applicable to Gravity Roller Conveyor, Fundamental and Applied Problems of Engineering and Technology, v. 342(4-1), pp. 106-116 (2020)

8. O.A. Ryakhovsky, S.S. Ivanov, Coupling Handbook (Politekhnika, St. Petersburg, 1991)

9. A.U. Ozolin, D.U. Skubov, L.V. Shtukin, Methods of Braking a Falling Elevator with the Help of Permanent Magnets, Scientific and Technical Journal of the St. Petersburg State Polytechnic University, v. 6(70), pp. 82-86 (2008) (in Russian)

10. O.A. Luskan, Determination of the Speed of Transportation of Parcel Goods on an Inertial Roller Conveyor, Izv. TulGU. Handling Machinery and Equipment, v. 4, pp. 84-89 (2003) (in Russian)

11. L. Ghomri, Z. Sari, Mathematical Modeling of the Average Retrieval Time for FlowRack Automated Storage and Retrieval Systems, J. Manuf. Syst., v. 44. pp. 165-178 (2017) 